\title{
RECOLECCIÓN DE LEGUMINOSAS FORRAJERAS NATIVAS Y DISTRIBUCIÓN NATURAL DE CENTROSEMA EN HONDURAS
}

\author{
Linus Wege ${ }^{1}$, Rainer Schultze-Kraft ${ }^{2}$, Conrado Burgos ${ }^{3}$
}

\begin{abstract}
RESUMEN
Recolección de leguminosas forrajeras nativas y distribución natural de Centrosema en Honduras. En febre-ro de 1989 se recolectó en Honduras germoplasma de leguminosas nativas con potencial forrajero. Se recorrieron 14 de 18 departamentos del país y se colectaron 427 muestras de semillas. Más del $80 \%$ corresponde, en orden descendente de número de muestras, a los géneros Centrosema, Desmodium, Aeschynomene, Stylosanthes, Vtgna, Macroptilium, Teramnus, Calopogonium, y Galactia. Se discute la distribución natural de las nueve especies de Centrosema nativas de Honduras y se presentan mapas de distribución. Se enfatiza la necesidad de misiones adicionales de recolección, principalmente en regiones de mayor peligro de erosión genética.
\end{abstract}

\begin{abstract}
Collection of native legumes with forage potential and natural distribution of Centrosema in Honduras. Gerrn plasm of native legumes with forage potential was collected in Honduras in February 1989. Four hundred and twenty-seven seed samples were taken from 14 out of the 18 departments of the country. More than $80 \%$ of fue samples corresponded in descending order of number of samples to the genuses Centrosema, Desmodium, Aeschynomene, Stylosanthes, Vtgna, Macroptilium, Teramnus, Calopogonium and Galactia. Natural distribution of the nine Centrosema species that were identified as native to Honduras is discussed, and distribution maps are presented. The need for additional missions to collect germ plasm, mainly from regions presenting a major danger for genetic erosion, is stressed.
\end{abstract}

\section{INTRODUCCIÓN}

En contraste con las plantas forrajeras de clima templado, el desarrollo de variedades de gramíneas y leguminosas forrajeras para regiones tropicales, se basa principalmente en poblaciones silvestres de especies tropicales de las cuales se seleccionan eco tipos con características deseables. Por lo tanto, la diversidad genética de la flora silvestre es la base para selecciones exitosas. Esta diversidad es muy grande en Africa tropical para la familia de las gramíneas y en América tropical para las leguminosas (Harlan, 1981).

En leguminosas tropicales con potencial forrajero, Centroamérica es uno de los centros de diversificación más importantes (Williams, 1983). Como tal, ha sido objeto de misiones de recolección de germoplasma; entre otros por parte de investigadores australianos, principalmente en la década de 1960. En esta época se co- lectó, por ejemplo, en Guatemala el material genético de Stylosanthes guianensis que después fue liberado en Australia como cv. Endeavour y en Costa Rica un ecotipo de Centrosema schiedeanum liberado en Australia en 1971 como cv. Belalto (Oram, 1990).

En Honduras, Nicaragua y El Salvador no se han efectuado actividades de recolección de germoplasma de leguminosas nativas. La Dirección General de Ganadería, Secretaría de Recursos Naturales de Honduras ha tenido interés en los recursos forrajeros nativos, pero sólo recientemente se han considerado las primeras actividades de recolección. Esto coincidió con la recomendación de la Quinta Reunión del Comité Asesor de la Red Internacional de Evaluación de Pastos Tropicales (RIEPT), celebrada en mayo 1987 en David, Panamá, de extender organizadamente las actividades de recolección de germoplasma a Centro américa como trabajo colaborativo entre el Centro Internacional de

\footnotetext{
1 Oberer Weg 2, D8901 Meitingen 1, Alemania.

2 Universidad Hohenheim (380), Postfach 700562, D - 7000 Stuttgart 70, Alemania.

3 Secretaría de Agricultura y Ganadería, Dirección de Ciencia y Tecnología Agropecuaria (DICTA) Tegucigalpa, MDC, Honduras.
} 
Agricultura Tropical (CIAT) en Cali, Colombia, y las instituciones nacionales. En forma paralela en la Reunión Internacional sobre Centrosema, celebrada en febrero 1987 en el CIAT, se recomendó dar prioridad a la recolección de germoplasma de este importante género en Centroamérica, particularmente las especies C. $m a$ crocarpum y $C$. schiedeanum.

Otras leguminosas de interés para la región son Desmanthus virgatus y Teramnus uncinatus. La primera se considera promisoria por su tolerancia a altas presiones de pastoreo bajo condiciones semiáridas ( $R$. Santillán, comunicación personal). A la segunda, según observaciones de poblaciones nativas en su hábitat silvestre, se le atribuye un buen potencial como forraje de reserva para la época seca y como mejorador de residuos de cosecha (L. Wege, información no publicada). En febrero de 1989 se llevó a cabo una misión de recolección de leguminosas nativas en Honduras, como proyecto colaborativo entre la Secretaría de Recursos Naturales de Honduras y el CIAT.

\section{MATERIALES Y MÉTODOS}

La recolección siguió la metodología descrita por Schultze-Kraft (1979). Esta consiste, en recorrer por carreteras o caminos un trayecto previamente determinado, haciendo paradas a intervalos de 20-50 km según los cambios que ocurran en la vegetación, la topografía y el suelo. En estas paradas se examina la vegetación nativa de un área adyacente o cercana a la carretera, que puede variar de unos pocos hasta varios miles de metros cuadrados y se buscan las especies que previamente se hayan señalado de interés. En este caso fueron las leguminosas en general, con énfasis en el género Centrosoma (nombre vulgar en Honduras: "Choreque"; subfamilia Papilionaceae), y en las especies Desmanthus virgatus (Mimosaceae) y Teramnus uncinatus (Papilionaceae).

En cada parada de 30-60 minutos, se recolecta la máxima cantidad posible de semillas del máximo número posible de plantas de una población. Las semillas se guardan en bolsas de papel debidamente identificadas. Cuando se trata de germoplasma considerado especialmente valioso pero del cual no se encuentran semillas maduras, se recolecta material vegetativo con raíces o con capacidad de enraizar. Este material se trasplanta posteriormente en macetas para multiplicar la semilla.

Se anotan las características particulares de las plantas muestreadas y se registra la información básica sobre el lugar de recolección (localización exacta, altitud, topografía, zona ecológica a la cual pertenece, hábitat y condiciones de suelo). En sitios escogidos se toman muestras de suelo para análisis y ocasionalmente se colectan nódulos de Rhizobium.
La misión de recolección tuvo lugar del 4 al 16 de febrero de 1989, con la participación de personal de la Secretaría de Recursos Naturales (Américo Rush Oscar Suazo y Linus Wege) y del CIAT (Rainer Shultze Kraft). Las semillas colectadas se dividieron en dos partes: Una quedó en la Dirección General de Ganadería, Secretaría de Recursos Naturales, con fines de evaluación; la otra se incorporó al Banco de Germoplasma del CIAT, para conservación y evaluación.

Durante el viaje se visitó el herbario "Paul C. Standley" de la Escuela Agrícola Panamericana (EAP) en el Zamorano, donde se revisó y en casos necesarios se reclasificó el material de Centrosema. Además se registraron los pormenores de los sitios de recolección de todas las muestras de Centrosema en la EAP, con excepción de C. pubescens, una especie muy frecuente en Honduras y de distribución amplia. Estos registros se combinaron con la información generada durante el viaje de recolección, para elaborar mapas de distribución natural de Centrosema spp. en Honduras. En la revisión del material de herbario de la EAP a veces la información en las etiquetas era tan deficiente que no fue posible la localización de los sitios de recolección en el mapa.

En la revisión del material de herbario de la EAP, un alto porcentaje de las muestras de Centrosema proviene de la región de El Zamorano y de zonas aledañas. Esto parece reflejar no tanto alguna particularidad respecto a la frecuencia de las especies en la zona sino más bien una exploración botánica intensiva en la vecindad de la EAP.

\section{RESULTADOS Y DISCUSIÓN}

\section{Recorrido}

Se recorrieron los departamentos de Francisco Morazán, El Paraíso, Olancho, Colón, Yoro, Atlántida, Cortez, Santa Bárbara, Copán, Lempira, Intibucá, Comayagua, Choluteca y Valle, a lo largo de una ruta de aproximadamente $2.500 \mathrm{~km}$ de largo, señalada en la Figura 1 . Se hicieron 82 paradas en las cuales se colectaron 427 muestras.

\section{Clima y Suelo}

Las recolecciones se hicieron en un amplio rango de condiciones climáticas que según el Atlas Climático de Honduras (Zúñiga, 1978) variaron de regiones semiáridas como en el sur del país o en los valles del interior con menos de $2000 \mathrm{~mm}$ de precipitación anual y ¡eis meses secos, hasta regiones de clima húmedo Figura 1. Ruta de recolección (LÍnea más gruesa) de leguminosas forrajeras en Honduras; febrero tropical con 


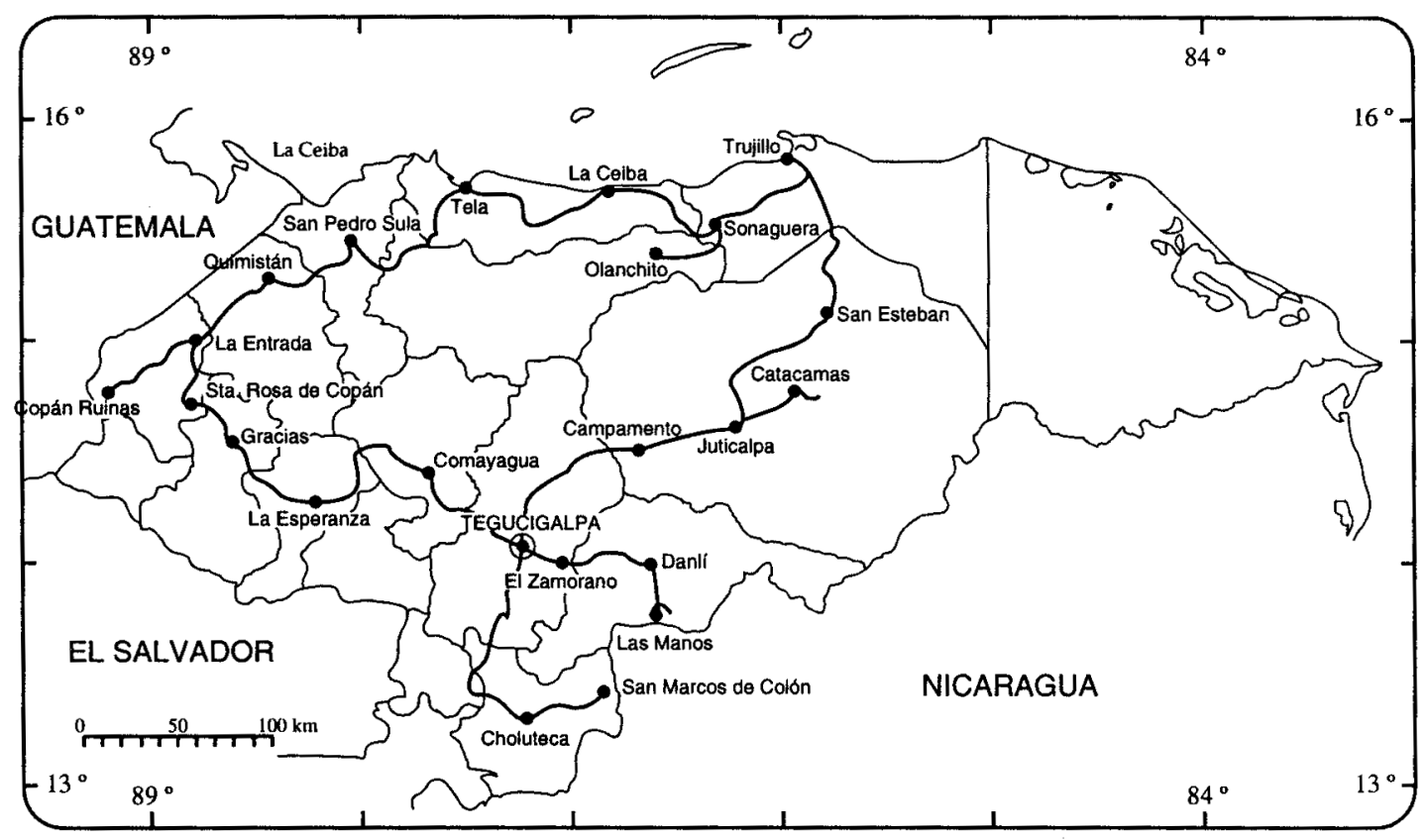

Figura 1. Ruta de recolección (Linea más gruesa) de leguminosas forrajeras en Honduras; febrero 1989.

2.600 - $3.400 \mathrm{~mm}$ de precipitación anual, 0-2 meses con precipitación inferior a $60 \mathrm{~mm}$ (zona de Litoral Atlántico). La mayor parte de las muestras se obtuvieron en clima subhúmedo característico para Honduras, con $1.000-2.000 \mathrm{~mm}$ de precipitación anual y 4-6 meses secos. Detalles climáticos, calculados teniendo como referencia la base de datos meteorológicos del CIAT, correspondientes a los sitios de muestreo están registrados en el reciente Catálogo de Germoplasma del CIAT (Schultze-Kraft, 1991).

En casi todos los sitios de recolección la capa arable del suelo tenía un espesor de $10-25 \mathrm{~cm}$, con una textura que variaba entre franco arenosa (aprox. 10\% de los sitios) y franco arcillosa. Especialmente en las regiones onduladas y montañosas los suelos eran de poca profundidad $(30-80 \mathrm{~cm})$. Tomando como referencia el potencial de producción agrícola (FAO, 1977), en más del $80 \%$ de los sitios la fertilidad natural se caracterizó como moderada. El pH de los suelos muestreados varió entre 5,1 y 6,6 .

\section{Germoplasma colectado}

De las 427 muestras de germoplasma colectadas (Cuadro 1), el género predominante fue Centrosema (19,4\%: 83 muestras), Desmodium (17,3\% : 74 muestras, principalmente $D$. incanum, D. barbatu, y D. intortum), Aeschynomene (9,4\% : 40 muestras, principalmente A. americana, A. brasiliana y A. falcata),
Stylosanthes $(8,4 \%$ : 36 muestras de $S$. guianensis, $S$. humilis, y una especie aún por determinar, posiblemente $S$. subsericea), Vigna, Macroptilium, Teramnus, $\mathrm{Ca}$ lopogonium, y Galactia $(7,0,5,6,5,2,4,7$ y 4,7\%, respectivamente). Algunas de las especies encontradas no eran nativas de Honduras sino naturalizadas (por ejemplo Flemingia strobilifera y Pueraria phaseoloides, ambas originarias del sudeste asiático).

\section{Germoplasma y distribución natural de Centrosema spp.}

C. pubescens Benth fue la especie más frecuente y de más amplia distribución natural en Honduras; se encontraba en casi todos los departamentos recorridos y en todas las zonas climáticas (Cuadro 2, Figura 2). La alta frecuencia quedó manifiesta también por la forma abundante en la cual $C$. pubescens está representada en el herbario EAP (los sitios de origen de las muestras de herbario no están incluidos en la Figura 2). Con las 32 muestras realizadas se aumentó en forma muy considerable la colección de germoplasma de la especie; hasta ahora sólo se disponía de dos ecotipos hondureños (IRFL 1719 y 1720 de los departamentos de Colón y Atlántida, respectivamente). El habitat más común de C. pubescens era matorrales, frecuentemente en zonas de carretera y bordes de caminos. La fertilidad del suelo en los sitios de recolección fue de mediana a alta; su textura varió entre franco arenoso, franco limosa y franco arcillosa. La especie forma también parte de los 
Cuadro 1. Resumen de germoplasma de leguminosas nativas colectadas en Honduras. Febrero de 1989.

\begin{tabular}{lclc}
\hline $\begin{array}{c}\text { Especies de } \\
\text { interes particular }\end{array}$ & $\begin{array}{c}\mathbf{N}^{\circ} \mathbf{d e} \\
\text { muestras }\end{array}$ & Otras especies & $\begin{array}{c}\mathbf{N}^{\circ} \text { de } \\
\text { muestras }\end{array}$ \\
\hline Centrosema & & Aeschynomene spp. & 40 \\
angustrifolium & 2 & Calliandra spp & 10 \\
macrocarpum & 10 & Calopogonium spp. & 20 \\
plumieri & 12 & Canavalia spp. & 12 \\
pubescens & 32 & Desmodium spp. & 74 \\
sagittatum & 10 & Galactia spp. & 20 \\
schiedeanum & 13 & Macroptilium spp. & 24 \\
schottii & 1 & Pachyrhizus spp. & 4 \\
virginianum & 3 & Phaseolus spp. & 11 \\
& & Rhynchosia spp. & 8 \\
Total Centrosema & 83 & Stylosanthes spp. & 36 \\
& & Vigna spp. & 30 \\
Desmanthus virgatus & 7 & Zonia spp. & 11 \\
Teramnus labials & 1 & Otros géneros* & 14 \\
Teramnus uncinatus & 21 & & \\
\hline
\end{tabular}

* Acacia (1 muestra), Alysicarpus (2), Chamaecrista (2), Crotalaria (1), Dioclea (1), Erythrina (1), Indigofera (1), Leucaena (2), Pueraria (2).

Cuadro 2. Ambitos de condiciones climáticas en sitios de recolección de muestras de Centrosema, Desmanthus y Teramnus en Honduras, febrero 1989.

\begin{tabular}{lcrrr}
\hline \multicolumn{1}{c}{ Especie } & $\begin{array}{c}\mathbf{N}^{\circ} \mathbf{d e} \\
\text { muestras }\end{array}$ & $\begin{array}{c}\text { Altitud } \\
(\mathbf{m s n m})\end{array}$ & $\begin{array}{c}\text { Precipitación } \\
(\mathbf{m m} / \mathbf{a n ̃})\end{array}$ & $\begin{array}{c}\text { Meses } \\
\text { secos* }\end{array}$ \\
\hline $\begin{array}{l}\text { Centrosema } \\
\text { angustifolium }\end{array}$ & 2 & & & \\
macrocarpum & 10 & $740-840$ & $1400-1800$ & 5 \\
plumieri & 12 & $20-1000$ & $1000-2800$ & $2-6$ \\
pubescens & 32 & $20-1000$ & $700-2800$ & $2-6$ \\
sagittatum & 10 & $310-900$ & $700-2000$ & $5-6$ \\
schiedeanum & 13 & $620-1320$ & $1000-1600$ & $5-6$ \\
schottii & 1 & 620 & $1200-1400$ & 6 \\
virginianum & 3 & $90-900$ & $1100-2000$ & $5-6$ \\
$\begin{array}{l}\text { Desmanthus } \\
\text { virgatus }\end{array}$ & 7 & $90-900$ & $700-2000$ & $5-6$ \\
Teramnus & & & & \\
libialis & & 900 & $1000-1200$ & 6 \\
uncinatus & 1 & $460-1320$ & $900-1600$ & $5-6$ \\
\hline
\end{tabular}

* Meses con precipitación $60 \mathrm{~mm}$.

pastizales en la zona del litoral atlántico donde la presión de pastoreo de baja a moderada (L. Wege, información no publicada).

En varios lugares $C$. pubescens estuvo asociada con C. macrocarpum y $C$. schiedeanum y en dos oca- siones se observaron plantas que evidentemente eran producto de hibridación natural entre estas especies. Esta observación confirma la relación genética muy estrecha entre las especies dentro del "complejo de Centrosema macrocarpum" (Williams y Clements, 1990). 


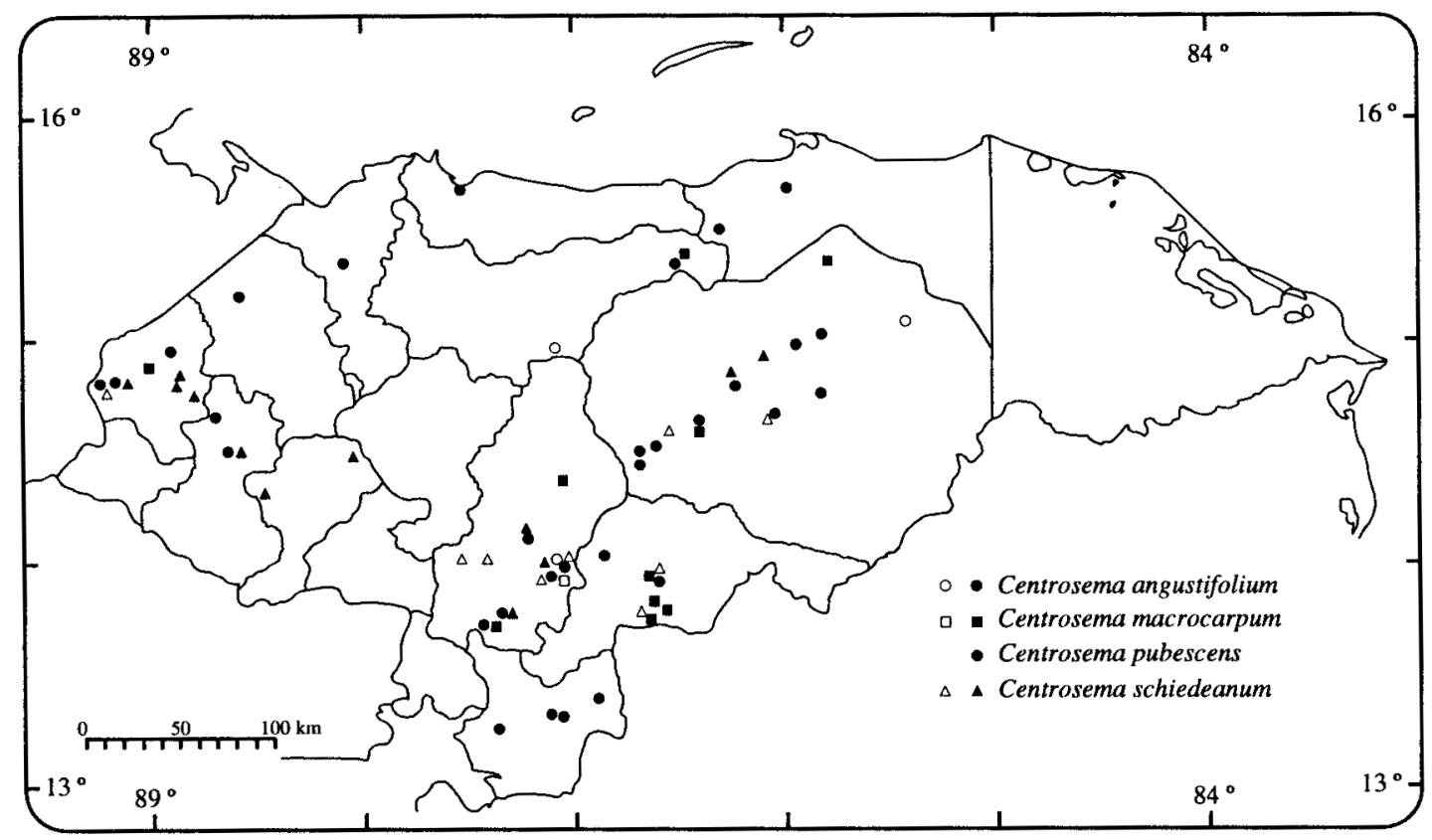

Figura 2. Distribución natural de Centrosema agustifolium, C. macrocarpum, C. pubescens y C. schiedeanum. en Honduras, 1989. Los símbolos cerrados se refieren a lugares de recolección de germoplasma (semilla).

Los símbolos abiertos a lugares de recolección de material herbario. Si en un lugar dado se dispone tanto de germoplasma como de material herbario, se da prioridad al símbolo de germoplasma. Un sólo símbolo puede referirse a más de una muestra de germoplasma o material herbario si la distanci.a entre los respectivos lugares de recolección es lo suficientemente corta.

Nota: Para C. pubescens se reportan sólo dos lugares de recolección de germoplasma.

C. schiedeanum Schlecht (13 muestras colectadas) fue la segunda especie más frecuente (Cuadro 2). En Honduras fue la primera vez que se colectó como germoplasma. Su distribución natural es también bastante amplia (Figura 2). Sin embargo, ocurre más que todo en las regiones de altura media del interior del país (620$1500 \mathrm{msnm}$ ) en bordes de bosques y como planta pionera, en zonas de apertura con suelos más bien fértiles. El material hondureño de $C$. shiedeanum es un tipo intermedio entre las formas mejicana y costarricensepanameña. La primera es similar a $C$. macrocaerpum, la segunda (por ejemplo representada por el cv. Belalto) es bastante similar a $C$. pubescens (Schultze-Kraft et al., 1990).

C. macrocarpum Benth (10 muestras colectadas) también resultó ser una especie más común en Honduras de lo que la información hasta ahora disponible (Schultze-Kraft et al., 1990) sugería (Figura 2). En su distribución natural se extiende a zonas mucho más lluviosas. La mayor parte de las muestras recolectadas (Cuadro 2) y del material de herbario examinado en la EAP, proviene de regiones subhúmedas con una marcada época seca. Fue la primera vez que se recolectó ger- moplasma de esta especie en Honduras. Tratándose de plantas enredaderas trepadoras generalmente muy vigorosas, el hábitat preferido de $C$. macrocarpum son matorrales y bordes de bosques donde encuentra el soporte necesario para enredarse.

C. angustifolim (H.B.K.) Benth, se recolectó en sólo dos lugares del departamento de Olancho (Figura 2) en clima subhúmedo (Cuadro 2), su hábitat se caracterizó por matorrales en zona de carretera sobre suelos arcillosos a franco arcillosos, de baja fertilidad y con drenaje deficiente. Aunque en la EAP se identificaron varias muestras de herbario como $C$. angustifolium, hasta ahora no se tenía conocimiento que esta especie creciera en Centroamérica, al norte de la latitud 10 grados (Schultze-Kraft et al., 1990).

C. plumieri (Turp. ex Pers.) Benth. sorprendió con 12 muestras recolectadas, pues hasta ahora no había registro de esta especie en Honduras en forma de material herbárico ni de germoplasma (Schultze-Kraft et al., 1990). Su distribución natural en Honduras parece ser bastante amplia (Figura 3), sobre todo en zonas de clima menos seco (Cuadro 2). Su hábitat preferido se ca- 


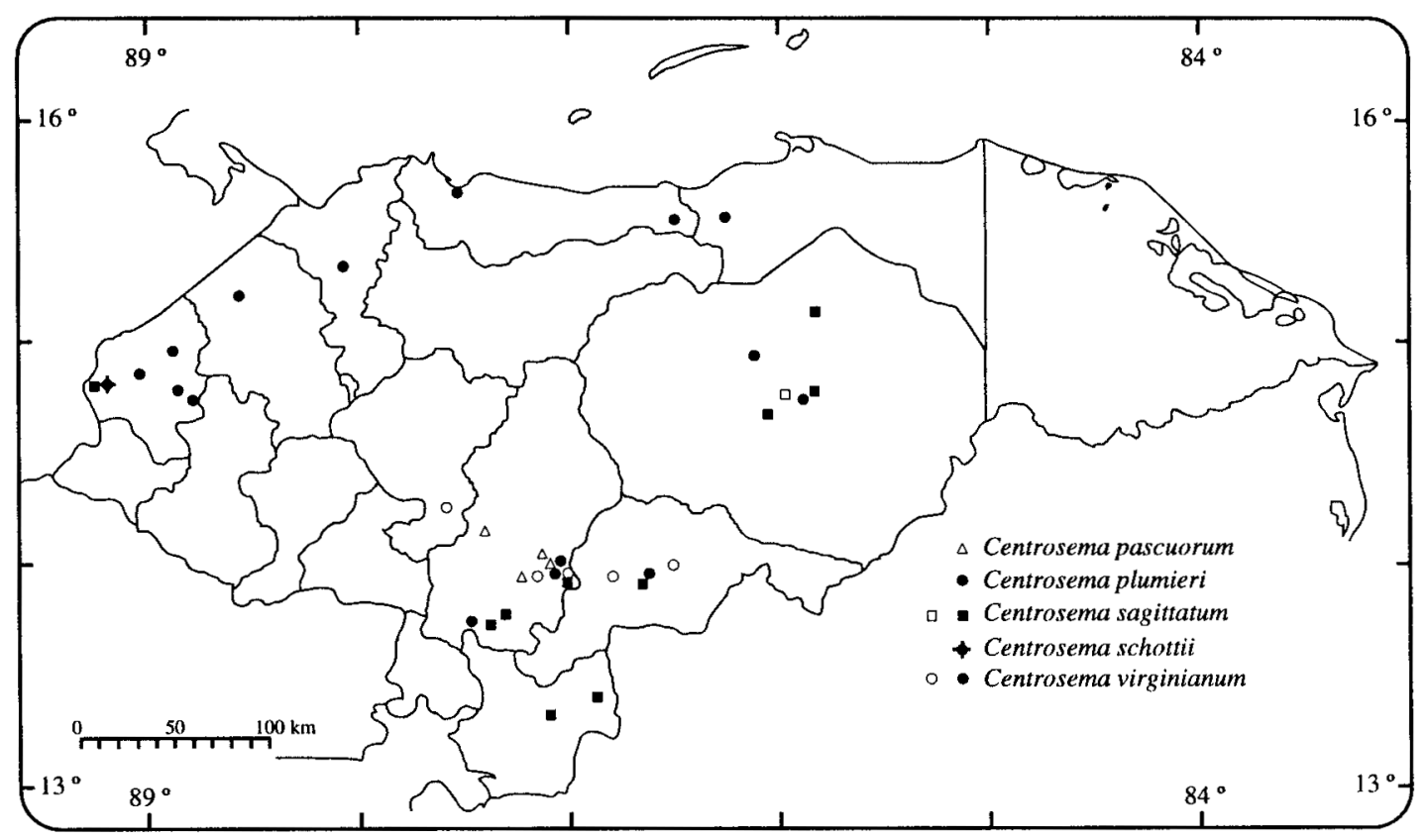

Figura 3. Distribución natural de Centrocema pascuorum, C. sagittatum, C. schottii y C. virginianum, C. plumieri en Honduras.

Los símbolos cerrados se refieren a lugares de recolección de germoplasma (semilla). Los símbolos abiertos a lugares de recolección de material herbario. Si en un lugar dado se dispone tanto de germoplasma como de material herbario, se da prioridad al símbolo de germoplasma. Un sólo símbolo puede referirse a más de una muestra de germoplasma o material herbario si la distancia entre los respectivos lugares de recolección es lo suficientemente corta.

racteriza por sitios algo húmedos y protegidos tales como matorrales de quebradas y en zonas de carretera; los suelos son generalmente de fertilidad mediana a alta.

C. sagittatum (H. \& B. ex Willd) Malme, una especie unifoliolada que en Honduras se conoce como "frijolillo" (Nelsons, 1986) se encontró también ampliamente distribuida (Figura 3), con tendencia hacia zonas menos lluviosas de cinco a seis meses secos (Cuadro 2).

Siendo una especie adaptada a ciertas condiciones de sombra, se encontró más que todo en matorrales densos donde se recolectaron 10 muestras en suelos de fertilidad mediana a alta.

C. virginianum Benth, que en Honduras se conoce por "amarratabaco" (Nelson, 1986), no es una especie muy común; con excepción de un lugar cerca de San Pedro Sula en el departamento de Cortés, su distribución natural parece estar restringida a la región central del país (Figura 3). Las tres muestras que se encontraron provienen de diferentes hábitats.
C. schottii (Millsp.) K. schum, es una especie anual-bianual, muy común en la parte mexicana de la Península de Yucatán, pero es la primera vez que se observa en Centroamérica (Figura 3, Cuadro 2). Se encontró una sola población de plantas que crecían a la salida de Copán Ruinas en un suelo calcáreo, franco arcilloso, de fertilidad mediana.

C. pascuorum Mart. ex Benth, es una especie de fructificación temprana y pronunciadamente anual de la cual no se encontraron plantas por lo avanzado de la época seca. Según la revisión del material de herbario en la EAP, C. pascuorum parece tener su distribución natural restringida a la región central del país, sobre todo en la zona de El Zamorano.

En aproximadamente $70 \%$ de los lugares, se encontró más de una especie de Centrosema. Las asociaciones más frecuentes fueron $C$. pubescens con $C$. sagittatum, C. pubescens con C. plumieri, C. pubescens con $C$. macrocarpum y pubescens con $C$. shieldeanum. Otra vez C. pubescens muestra su gran adaptación a diferentes condiciones ecológicas. 


\section{Conclusiones y recomendaciones sobre Desmanthus virgatus y Teramnus uncinatus}

Desmanthus virgatus (L.) Willd. (siete muestras colectadas) se encontró en lugares con períodos de sequía de cinco a seis meses y en suelos franco arcillosos hasta arcillosos, frecuentemente con drenaje deficiente. Las muestras colectadas provienen de zonas de altura mediana en el interior del país.

Teramnus uncinatus (L.) Swartz (21 muestras colectadas) es una especie frecuente en bordes de bosques, sobre todo en las zonas de altura mediana del interior. Prefiere suelos franco limosos a franco arcillosos, de fertilidad mediana a alta.

La primer misión de recolección sistemática en Honduras obtuvo una gran cantidad de leguminosas nativas. Las muestras colectadas son representativas de la vegetación adaptada a las más diversas condiciones ecológicas, particularmente con respecto al clima. En contraste con misiones de recolección similares en Panamá (Duque, et al., 1986) y México (Peralta et al., 1987), los suelos no resultaron muy pobres; un indicio para ello es también el alto porcentaje de especies y géneros colectados que como Centrosema pubescens, Aeschynomene, Calopogonium, Galactia, Macroptilium, Teramnus y Vigna, no son plantas indicadoras de suelos de baja fertilidad.

La recolección sistemática de germoplasma de leguminosas en Honduras, se debió haber extendido hasta regiones no cubiertas. Las zonas de mayor desarrollo agrícola deberían recibir atención debido al peligro de erosión genética.

Respecto al género Centrosema se ha completado en gran medida los conocimientos sobre las especies nativas de Honduras y su distribución. La poca información que hasta ahora se tenía sobre algunas especies, se originó en la identificación de las especies y, por lo tanto, con el uso incorrecto de nombres.

El germoplasma colectado de Centrosema spp. no sólo será útil para futuros trabajos de desarrollo de variedades forrajeras mediante evaluación y selección, o en proyectos de fitomejoramiento, sino también contribuirá a estudios citotaxonómicos y quimotaxonómicos para elucidar las relaciones interespecíficas, sobre todo dentro de grupos de especies muy afines como C. macrocarpum, $C$. pubescens y $C$. chiedeanum.

\section{LITERATURA CITADA}

DUQUE, O.; ARGEL, P.J.; SCHULTZE-KRAFT, R. 1986. Recolección de germoplasma nativo de leguminosas forrajeras en Panamá. Pasturas Tropicales, Boletín 8 (1): 10-14.

FAO, 1977. Informe al gobierno de Honduras sobre los suelos de Honduras. FAO, Roma, Italia, 92 p.

HARLAN, J.R 1981. Use of genetic resources for improvement of forage species. In: Smith, J.A. y V.M. Hays (eds.). Proceedings, 14th International Grassland Congress, Lexington, Kentucky. Westview Press, Bou1der, Colorado, USA. pp. 29-34.

NELSON SUTHERLAND, C.H. 1986. Plantas comunes de Honduras. Universidad Nacional Autónoma de Honduras, Tegucigalpa, Honduras, 922 p.

ORAM, RN. (comp.) 1990. Register of Australian herbage plant cultivars. 3rd. de. CSIRO, Me1bourne, Australia. $304 \mathrm{p}$.

PERALTA, A.; SCHULTZE-KRAFT, R.; MARTINEZ, J.M.; AGUIRRE, J.F.; AMAYA. H.S.; ENRIQUEZ, J. 1987. Recolección de leguminosas forrajeras nativas en el trópico de México. Pasturas tropicales-boletín 9 (1): 21-26.

SCHULTZE- KRAFT, R 1979. Colección de germoplasma en el campo. In: Mott, G.O. (ed.), Manual para la colección, preservación y caracterización de recursos forrajeros tropicales. CIAT, Cali, Colombia, pp. 9-14.

SCHULTZE-KRAFT, R 1991. La colección de forrajeras tropicales del CIAT. 3 Catálogo de Germoplasma de Centroamérica, México y el Caribe. Documento de Trabajo N'90, CIAT, Cali, Colombia. 269 p.

SCHULTZE-KRAFT, R; WILLIAMS, L. CORADIN. 1990. Biogeography of Centrosema. In: Schultze-Kraft, R.; R J. C1ements (eds.), Centrosema: Biology, agronomy and utilizaton. CIAT, Cali, Colombia, pp. 29-76.

WILLIAMS, R.J. 1983. Tropica11egumes. In: McIvor, J.G. y R.A. Bray (eds.), Genetic resources of forage plants. CSIRO, Melbourne, Australia, pp. 17-37.

WILLIAMS, R.J.; R.J. CLEMENTS. 1990. Taxonomy of Centrosema. In: Schu1tze-Kraft, R y R.J. Clements (eds.) Cali, Colombia, pp. 1-27.

ZUÑIGA, A.E. 1978. Isohetas medias en milímetros. In: Ministerio de Recursos Naturales (ed.). Atlas Climático de Honduras. Tegucigalpa, Honduras, 24 p. 
